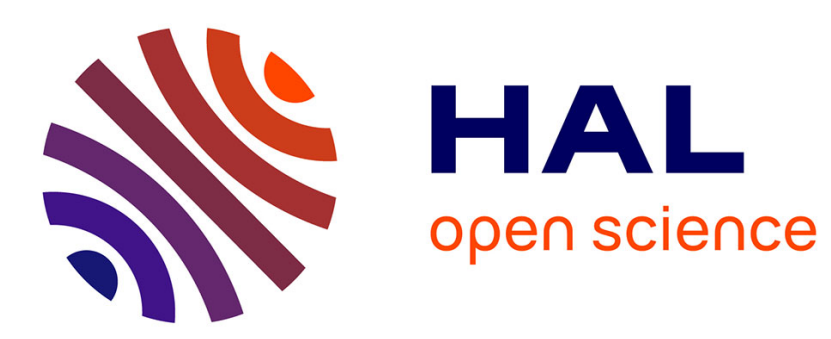

\title{
Existence and uniqueness theorems in two-dimensional nematodynamics. Finite speed of propagation
}

\author{
T. S. Ratiu, M. S. Romanov, V. N. Samokhin, G. A. Chechkin
}

\section{To cite this version:}

T. S. Ratiu, M. S. Romanov, V. N. Samokhin, G. A. Chechkin. Existence and uniqueness theorems in two-dimensional nematodynamics. Finite speed of propagation.

Mathematics, 2015, 91 (3), pp.354-358. hal-01398390

/ Doklady

\section{HAL Id: hal-01398390 \\ https://hal.science/hal-01398390}

Submitted on 17 Nov 2016

HAL is a multi-disciplinary open access archive for the deposit and dissemination of scientific research documents, whether they are published or not. The documents may come from teaching and research institutions in France or abroad, or from public or private research centers.
L'archive ouverte pluridisciplinaire HAL, est destinée au dépôt et à la diffusion de documents scientifiques de niveau recherche, publiés ou non, émanant des établissements d'enseignement et de recherche français ou étrangers, des laboratoires publics ou privés. 


\title{
Existence and Uniqueness Theorems in Two-Dimensional Nematodynamics. Finite Speed of Propagation ${ }^{1}$
}

\author{
T. S. Ratiu ${ }^{b}$, M. S. Romanov ${ }^{a}$, V. N. Samokhin ${ }^{c}$, and G. A. Chechkin ${ }^{a}$ \\ Presented by Academician V. V. Kozlov December 3, 2014
}

\begin{abstract}
The paper is devoted to the two-dimensional Ericksen-Leslie system describing the nematodinamics of liquid crystals. The moment of inertia of molecules is supposed to be strictly positive. The existence of the solution was proved in the case of periodic domain and in the case of bounded domain. In the last case media is supposed to adhere to the solid surface, the director vector field describing orientation of the molecules is constant in the neighbourhood of the boundary. The uniqueness of the strong solution was proved in both cases. Also we prove the propagation of director disturbance has finite speed. This fact shows the difference between the model under consideration and models with zero moment of inertia of the molecules. The estimate of the speed of propagation depending on physical properties of the liquid crystal and the flow was obtained.
\end{abstract}

\section{INTRODUCTION}

Liquid crystals were discovered by the botanical physiologist Friedrich Reinitzer in 1888. In 1900 liquid crystals were first studied by the physicist Otto Lehman. Around the same time chemist Daniel Vorländer synthesized most of the liquid crystal types known at the moment and the mineralogist and crystallographer George Friedel gave a first classification of the liquid crystals. The first physical theory of liquid crystals was formulated during the 1930 s by the physicist Carl Wilhelm Oseen. Later the physicist V.N. Tsvetkov used viscosity, dielectric, and diamagnetic anisotropy of liquid crystals to create a general theory of the mesomorphic state leading to the understanding of the dynamics of the mesophases.

In this report we study the existense and uniqueness of the solutions to the Ericksen-Leslie system, describing hydrodinamics of nematic liquid crystals.

The state of the liquid crystal is characterized by velocity vector of the media as well as director vector describing the orientation of molecules. In the case of the nematic calamitic liquid crystals (elongated

\footnotetext{
${ }^{1}$ The article was translated by the authors.

${ }^{a}$ Faculty of Mechanics and Mathematics, Moscow State University, Moscow, Russia

${ }^{b}$ École Polytechnique Fédérale de Lausanne, Switzerland

${ }^{c}$ Moscow State University of Printing Art, Moscow, Russia e-mail:mcliz@mail.ru
}

molecular dipoles) the director vector is colinear to the molecule's axis and to the velocity vector. In the case of nematic discotic liquid crystals the director is orthogonal to the velocity (see Fig. 1).

Since the structure of the full Ericksen-Leslie system is sufficiently complicated, the preceding papers dealt with essentially simplified models (see, e.g., [1-5]). On the contrary, we consider the system with minimal natural simplification (see below) and suppose the moment of inertia of the molecule to be non-zero. This assumption makes the problem more complex since the equation has second order time-derivative.

In our previous papers we studied periodic mesomorphic medium [6] and dinamics of micro inhomogenious nematic liquid crystals using the homogenization methods [7].

The subject of our research is the Ericksen-Leslie system $($ see $[8,9])$

$$
\begin{gathered}
\dot{\mathbf{u}}-\mu \Delta \mathbf{u}=-\nabla p-\frac{\partial}{\partial x_{j}}\left(\frac{\partial \mathscr{F}}{\partial \mathbf{n}_{x_{j}}} \cdot \nabla \mathbf{n}\right)+\mathbf{F}+f, \\
\operatorname{div} \mathbf{u}=0, \\
J \ddot{\mathbf{n}}-2 q \mathbf{n}+\mathbf{h}=g+\mathbf{G}, \quad\|\mathbf{n}\|=1,
\end{gathered}
$$

where summation on repeated indices is understood and $n_{x_{j}}:=\frac{\partial}{\partial x_{j}} \mathbf{n}$. Here, $\mathbf{u}$ is the spatial velocity vector field, $\mathbf{n}$ is the director field, $\mu>0$ is the viscosity coefficient, $J>0$ is the moment of inertia of the molecule, 
$\mathbf{F}(x, t)$ and $\mathbf{G}(x, t)$ are given external forces, and $:=\frac{\partial}{\partial t}+$ $\mathbf{u} \cdot \nabla$ is the material derivative. The crystal's density is supposed to be equal to one. The terms $f$ and $g$ correspond to the dissipative part of the stress tensor and the dissipative part of the intrinsic body force respectively, and they depend on $\mathbf{u}, \mathbf{n}$, and their derivatives. The function $\mathscr{F}(\mathbf{n}, \nabla \mathbf{n})$ is called the Oseen-Zöcher-Frank free energy and is defined by the formula

$$
\begin{aligned}
& \mathscr{F}(\mathbf{n}, \nabla \mathbf{n}):=K_{1} \mathbf{n} \cdot \operatorname{curl} \mathbf{n}+\frac{1}{2}\left(K_{11}(\operatorname{div} \mathbf{n})^{2}\right. \\
& \left.\quad+K_{22}(\mathbf{n} \cdot \operatorname{curl} \mathbf{n})^{2}+K_{33}\|\mathbf{n} \times \operatorname{curl} \mathbf{n}\|^{2}\right) .
\end{aligned}
$$

The molecular field is defined by $\mathbf{h}:=\frac{\partial \mathscr{F}}{\partial \mathbf{n}}-$ $\frac{\partial}{\partial x_{j}}\left(\frac{\partial \mathscr{F}}{\partial \mathbf{n}_{x_{j}}}\right)$

The pressure $p$ and the Lagrange multiplier $2 q$ are determined, respectively, by the conditions $\operatorname{div} \mathbf{u}=0$ and $\|\mathbf{n}\|=1$.

We are interested in the non-dissipative case, i.e., $g=0, f=0$. Since the liquid crystal is nematic, we necessarily have $K_{1}=0$. For simplicity, we study the case $K_{11}=K_{22}=K_{33}=: K>0$.

With all these hypotheses, system (1) becomes

$$
\begin{gathered}
\dot{\mathbf{u}}-\mu \Delta \mathbf{u}=-\nabla p-\left(K \mathbf{n}_{x_{j}} \cdot \nabla \mathbf{n}\right)_{x_{j}}+\mathbf{F}, \quad \operatorname{div} \mathbf{u}=0, \\
J \dot{\mathbf{v}}=-K \Delta \mathbf{n} \times \mathbf{n}+\mathbf{n} \times \mathbf{G}, \\
\dot{\mathbf{n}}=\mathbf{v} \times \mathbf{n},
\end{gathered}
$$

with unknowns $\mathbf{u}, \mathbf{v}, \mathbf{n}$, where $\mathbf{v}$ is a new vector field introduced in [10] (see also [11]) and the index $x_{j}$ means partial derivative relative to the spatial coordinate $x_{j}$.

Conversely, if the initial conditions of the Ericksen-Leslie system (1) for $t=0$ satisfy the identities

$$
\|\mathbf{n}(x, 0)\|=1, \quad \mathbf{n}(x, 0) \perp \mathbf{v}(x, 0),
$$

then for any $t>0$ we have

$$
\|\mathbf{n}\| \equiv 1, \quad \mathbf{v}=\mathbf{n} \times \dot{\mathbf{n}}, \quad 2 q=\mathbf{n} \cdot \mathbf{h}-J\|\mathbf{v}\|^{2},
$$

and (2)-(4) turns into (1). Thus, under these hypotheses on the initial conditions the systems (1) and (2)(4) are equivalent.

In the present paper we prove an existence and uniqueness theorems for solutions to the system (2)(4) in the case of two-dimensional periodic media as well as for the problem in bounded domain of the plane. We also prove the finite propagation speed of waves in such media. (a)

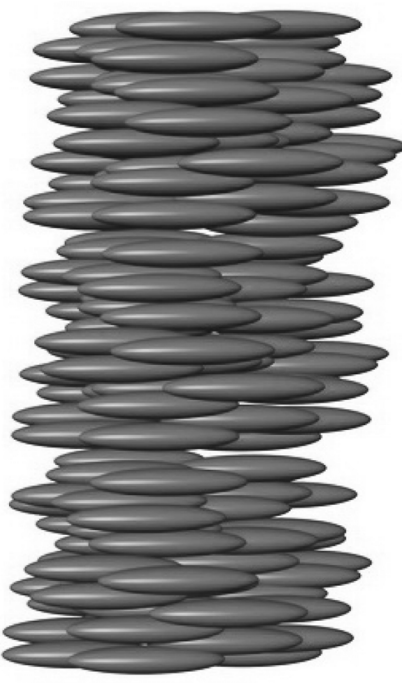

(b)

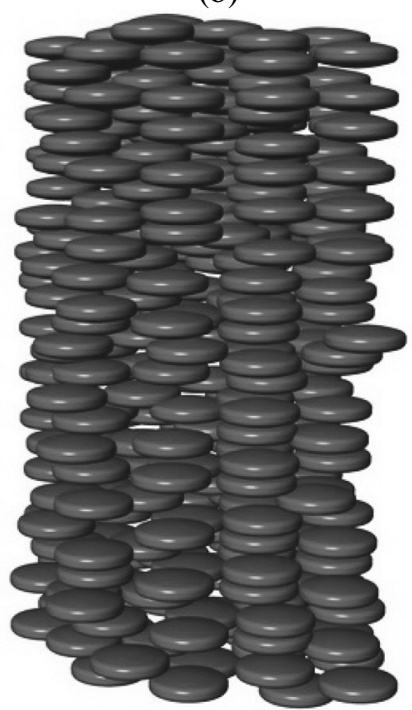

Fig. 1. The structure of nematic calamitic (a), and nematic discotic liquid crystals (b).

\section{TWO-DIMENSIONAL SOLUTION IN A PERIODIC DOMAIN}

Consider a nematic flow in $\mathbb{R}^{3}$. The flow is called two-dimensional if all unknowns in the EricksenLeslie system are independent of the third coordinate $x_{3}$; so we can suppose that they are all defined on a plane $\left(x_{1}, x_{2}\right)$.

Let $Q_{T}:=(0, T) \times \mathbb{Z}$, where $\mathbb{R}^{2} / \mathbb{Z}^{2}$ is the twodimensional flat torus.

We shall study the system (2)-(4) in $Q_{T}$ with initial conditions

$$
\mathbf{u}(0, x)=\mathbf{u}_{0}, \quad \mathbf{v}(0, x)=\mathbf{v}_{0}, \quad \mathbf{n}(0, x)=\mathbf{n}_{0} .
$$

Here $\mathbf{u}, \mathbf{v}, \mathbf{n}$ are unknown vector fields, $p$ is an unknown scalar function, and $J, K, \mu$ are fixed strictly positive numbers. The material derivative $f_{t}+\sum_{i=1}^{3} u^{i} f_{x_{i}}$ turns into $f_{t}+\sum_{i=1}^{2} u^{i} f_{x_{i}}$.

It is natural to suppose that $\mathbf{u}: \mathbb{\mathbb { T }} \rightarrow \mathbb{R}^{2} \times\left\{u^{3}=0\right\}$. Note that the vector fields $\mathbf{n}, \mathbf{v}$ are still three-dimensional, even if they are defined on a flat two-dimensional domain; in particular the director field is not necessarily tangential to the plane $\left\{x_{3}=0\right\}$.

Throughout the paper we use the following notations:

$$
L_{2}(\mathbb{\mathbb { V }}):=\left\{\mathbf{v}: \mathbb{T} \rightarrow \mathbb{R}^{3} \mid \int_{\mathbb{T}}\|\mathbf{v}\|^{2} d x<\infty\right\} ; \operatorname{Sol}(\mathbb{\mathbb { T }}):=\{\mathbf{v}:
$$

$\left.\mathbb{T} \rightarrow \mathbb{R}^{3} \mid \mathbf{v} \in \mathrm{C}^{\infty}(\mathbb{\mathbb { V }}), \operatorname{div} \mathbf{v}=0\right\} ; \operatorname{Sol}\left(Q_{T}\right):=\left\{\mathbf{v} \in C^{\infty}\left(Q_{T}\right) \mid\right.$ $\mathbf{v}(t, \cdot) \in \operatorname{Sol}(\mathbb{\mathbb { T }}), \forall t \in(0, T)\} ; \operatorname{Sol}_{2}(\mathbb{\mathbb { T }})$ is the closure of 
$\operatorname{Sol}(\mathbb{T})$ in the norm $L_{2}(\mathbb{\mathbb { V }})$; $\operatorname{Sol}_{2}^{m}(\mathbb{\mathbb { T }})$ is the closure of Sol( $\mathbb{T})$ in the norm $W_{2}^{m}(\mathbb{T})$.

Definition 1. A quadruple $(\mathbf{u}, \mathbf{v}, \mathbf{n}, p)$ is a strong solution of problem (2)-(5) in the domain $Q_{T}$, if $\mathbf{u} \in$ $L_{2}\left((0, T) ; \operatorname{Sol}_{2}^{3}(\mathbb{\mathbb { T }})\right), \mathbf{u}_{t} \in L_{2}\left(Q_{T}\right) ; \mathbf{v} \in L_{\infty}((0, T) ;$ $\left.W_{2}^{2}(\mathbb{T})\right), \mathbf{v}_{t} \in L_{\infty}\left((0, T) ; L_{2}(\mathbb{T})\right) ; \mathbf{n} \in L_{\infty}((0, T) ;$ $\left.W_{2}^{3}(\mathbb{T})\right), \mathbf{n}_{t} \in L_{\infty}\left((0, T) ; W_{2}^{1}(\mathbb{T})\right) ; \nabla p \in L_{2}\left(Q_{T}\right) ; \mathbf{u}, \mathbf{n}, \mathbf{v}$ satisfy the initial conditions (5), i.e. $(\mathbf{u}, \mathbf{n}, \mathbf{v}) \rightarrow\left(\mathbf{u}_{0}, \mathbf{n}_{0}, \mathbf{v}_{0}\right)$ weakly in $L_{2}(\mathbb{T})$ as $t \rightarrow 0$, Eqs. (2)-(4) hold almost everywhere.

The following theorems holds.

Theorem 1. Suppose $\mathbf{u}_{0} \in \operatorname{Sol}_{2}^{2}(\mathbb{T}), \mathbf{v}_{0} \in W_{2}^{2}(\mathbb{T})$, $\mathbf{n}_{0} \in W_{2}^{3}(\mathbb{\mathbb { T }})$ and $\mathbf{F} \in L_{2}\left((0, T) ; W_{2}^{1}(\mathbb{\mathbb { T }})\right), \mathbf{G} \in L_{1}((0, T)$; $\left.W_{2}^{2}(\mathbb{T})\right) ; F^{3}=0$. Then there exists the solution to problem (2)-(4) on the interval $(0, T)$ for some $T>0$.

Theorem 2. Suppose $\left(\mathbf{u}_{1}, \mathbf{v}_{1}, \mathbf{n}_{1}, p_{1}\right)$ and $\left(\mathbf{u}_{2}, \mathbf{v}_{2}\right.$, $\left.\mathbf{n}_{2}, p_{2}\right)$ to be the solutions to the problem (2)-(5) in the domain $Q_{T}$. Then for som $T_{0}$ : $0<T_{0} \leq T$ one has the equation

$$
\left(\mathbf{u}_{2}, \mathbf{v}_{2}, \mathbf{n}_{2}, \nabla p_{2}\right)=\left(\mathbf{u}_{1}, \mathbf{v}_{1}, \mathbf{n}_{1}, \nabla p_{1}\right)
$$

almost everywhere on $Q_{T_{0}}$.

\section{LIQUID CRYSTAL FLOW IN BOUNDED DOMAINS}

Let $\Omega$ be a bounded domain in $\mathbb{R}^{2}$. Consider nematic liquid crystal flow which does not depend on the third coordinate in the cylinder $\Omega \times \mathbb{R}$.

Since all functions in the Ericksen-Leslie system depend only on the points $\left(x_{1}, x_{2}\right) \in \Omega$, we are studying Eqs. (2)-(4) in domain $(0, T) \times \Omega$ with initial conditions (5) and additional boundary conditions

$$
\begin{gathered}
\left.\mathbf{u}\right|_{\partial \Omega}=0, \quad \mathbf{n}-\left.\mathfrak{i}\right|_{\partial \Omega}=0, \\
\left.\mathbf{v}\right|_{\partial \Omega}=0 \text { for any } t>0,
\end{gathered}
$$

where $\mathfrak{l}$ is a given vector field on $\Omega \times \mathbb{R}$.

Condition $\left.\mathbf{u}\right|_{\partial \Omega}=0$ means that the domain has impenetrable boundary and that the fluid moves without slipping; $\mathbf{n}-\left.\mathfrak{N}\right|_{\partial \Omega}=0$ describes the director position at the boundary. The third condition comes from the original Ericksen-Leslie system and means that $\dot{\mathbf{n}}=0$ at the boundary.

In this section we suppose $Q_{T}:=(0, T) \times \Omega$, So $(\Omega):=$ $\left\{\mathbf{v}: \Omega \rightarrow \mathbb{R}^{3}\right.$ such that $\left.\mathbf{v} \in C_{0}^{\infty}(\Omega), \operatorname{div} \mathbf{v}=0\right\} ; \operatorname{Sol}\left(Q_{T}\right):=$ $\left\{\mathbf{v} \in C^{\infty}\left(Q_{T}\right): \forall t \mathbf{v}(t, \cdot) \in \stackrel{\circ}{\operatorname{Sol}}(\Omega)\right\} ; \operatorname{Sol}_{2}^{m}(\Omega)$ is the closure of $\stackrel{\circ}{\mathrm{Sol}}(\Omega)$ in the norm $W_{2}^{m}(\Omega) ; \stackrel{\circ}{W}_{2}^{m}(\Omega)$ is the subspace of $W_{2}^{m}(\Omega)$ with zero trace (see, for instance $[13,14]$ ).

The definition of a solution of the Ericksen-Leslie equations is quite similar to the one in Definition 1, with few changes because of the boundary.

Definition 2. The quadruple ( $\mathbf{u}, \mathbf{v}, \mathbf{n}, p)$ is a strong solution of problem (2)-(5), (6) in domain $Q_{T}$, if $\mathbf{u} \in$ $L_{2}\left((0, T) ; \quad \operatorname{Sol}_{2}^{1}(\Omega)\right) \cap L_{2}\left((0, T) ; \quad W_{2}^{2}(\Omega)\right), \quad \mathbf{u}_{t} \in$ $L_{2}\left(Q_{T}\right) ; \mathbf{v} \in L_{\infty}\left((0, T) ; \stackrel{\circ}{W}_{2}^{1}(\Omega)\right), \mathbf{v}_{t} \in L_{\infty}((0, T)$; $\left.L_{2}(\Omega)\right)$, where $(\mathbf{n}-\mathfrak{l})$ is a vector field in $L_{\infty}((0, T)$; $\left.\stackrel{\circ}{W}_{2}^{l}(\Omega)\right) \cap L_{2}\left((0, T) ; W_{2}^{2}(\Omega)\right)$, where $\mathfrak{i}(x, t)$ is a given constant vector field, $\mathbf{n}_{t} \in L_{\infty}\left((0, T) ; W_{2}^{1}(\Omega)\right)$; $\nabla p \in L_{2}\left(Q_{T}\right) ; \mathbf{u}, \mathbf{n}, \mathbf{v}$ satisfy initial conditions (5), i.e., $(\mathbf{u}, \mathbf{n}, \mathbf{v}) \rightarrow\left(\mathbf{u}_{0}, \mathbf{n}_{0}, \mathbf{v}_{0}\right)$ weakly in $L_{2}(\Omega)$ and Eqs. (2)-(4) hold almost everywhere.

In this section we suppose the third component of the director to be equal to zero. Then we have

$$
\mathbf{n}=(\cos \theta, \sin \theta, 0), \quad \mathbf{v}=(0,0, v),
$$

where $\theta$ is a new unknown function. The EricksenLeslie system becomes

$$
\begin{gathered}
\dot{\mathbf{u}}-\mu \Delta \mathbf{u}=-\nabla\left(p+\frac{K}{2}\|\nabla \theta\|^{2}\right)-K \Delta \theta \nabla \theta+\mathbf{F}, \\
\operatorname{div} \mathbf{u}=0, \\
J \dot{\mathbf{v}}=-K \Delta \theta, \\
\dot{\theta}=\mathbf{v}
\end{gathered}
$$

with boundary and initial conditions

$$
\begin{gathered}
\left.\mathbf{u}\right|_{\partial \Omega}=0, \quad \theta-\left.\theta_{1}\right|_{\partial \Omega}=0, \\
\left.\mathbf{v}\right|_{\partial \Omega}=0 \text { for any } t>0, \\
\mathbf{u}(0, x)=\mathbf{u}_{0}(x), \quad \mathbf{v}(0, x)=v_{0}(x), \\
\theta(0, x)=\theta_{0}(x) .
\end{gathered}
$$

We have

Theorem 3. Assume that $\Omega$ is a convex Lipschitz domain and for almost all $x \in \partial \Omega$ the boundary is a graph of a $C^{2}$-function in some neighbourhood of $x$. Let $\theta_{0} \in W_{2}^{3}(\Omega), \mathbf{v}_{0} \in W_{2}^{2}(\Omega), \mathbf{u}_{0} \in \operatorname{Sol}_{2}^{1}(\Omega) \cap W_{2}^{2}(\Omega) ;$ $\left.\Delta \mathbf{u}_{0}\right|_{\partial \Omega}=0$ and assume that for some $d>0$ we have

$$
\begin{gathered}
\theta_{0}(x)=\theta_{1} \equiv \text { const, } \quad v_{0}(x)=0, \\
\text { if } \operatorname{dist}(x, \partial \Omega)<d,
\end{gathered}
$$

$\mathbf{F} \in L_{2}\left((0, T) ; W_{2}^{1}(\Omega)\right), \mathbf{G}=\left(G_{1}, G_{2}, 0\right) \in L_{1}((0, T) ;$ $\left.W_{2}^{2}(\Omega)\right), F^{3}=0$. Then the solution exists and is unique for some $T>0$. 
Remark 1. The analogues of the Theorems 3 hold also in the case of the director field

$$
\begin{gathered}
\mathbf{n}=\left(\cos \theta \sin \varphi_{0}, \sin \theta \sin \varphi_{0}, \cos \varphi_{0}\right), \\
\mathbf{v}=\left(0,0, v_{3}\right),
\end{gathered}
$$

where $\varphi_{0}$ is a constant angle and $\theta, v_{3}$ are new unknown functions.

\section{FINITE PROPAGATION SPEED}

In this section we consider the strong solutions of the Ericksen-Leslie equation both in the periodic (see Definition 1) and the bounded domain case (see Definition 2). For simplicity in this section we will use

$$
\int f d x_{1} d x_{2} \text { instead of } \int_{\mathbb{T}} f d x_{1} d x_{2} \text { or } \int_{\Omega} f d x_{1} d x_{2},
$$

depending on which domain we are working.

Denote $w^{i j}:=u_{x_{i}}^{j}+u_{x_{j}}^{i}$.

Theorem 4. Consider the Eqs. (3), (4), suppose that for some for some $1<\alpha \leq \infty$,

$$
\| \text { ess } \sup _{x}\left|w^{i j}(x, t)\right| \|_{L_{\alpha}(0, T)} \leq \frac{M}{2}
$$

and that $\|\mathbf{u}\|$ is bounded by a constant $m>0$. Assume also that $\nabla \mathbf{n}_{0}$ and $\mathbf{v}_{0}$ vanish for $\left\|x-x_{0}\right\|<r$. Then $\nabla \mathbf{n}$ and $\mathbf{v}$ are equal to zero for

$$
\left\|x-x_{0}\right\|<r-\left(m+\max \left\{1, \frac{K}{J}\right\}\right) t, \quad M t^{\frac{\alpha-1}{\alpha}}<\frac{1}{2} .
$$

In the case of a bounded domain $\Omega$, we impose the additional assumption $\mathbf{u}_{\partial \Omega}=0, \mathbf{v}(x, t)=0, \mathbf{n}(x, t)_{t}=0$, if $(x, t) \in\left\{x \in \partial \Omega,\left\|x-x_{0}\right\|<r-t(m+\right.$ $\left.\left.\max \left\{1, \frac{K}{J}\right\}\right)\right\}$

Proof. Let $x_{0}=0$. Using integral identities for the solutions of problem (3), (4) we get

$$
\begin{gathered}
\int\left(\frac{J}{K} \dot{\mathbf{v}} \cdot \zeta+(\Delta \mathbf{n} \times \mathbf{n}) \cdot \zeta\right) d x_{1} d x_{2} d t=0, \\
\int(\dot{\mathbf{n}} \cdot \psi-(\mathbf{v} \times \mathbf{n}) \cdot \psi) d x_{1} d x_{2} d t=0 .
\end{gathered}
$$

Let test functions be $\zeta=\mathbf{v} \varphi$ and $\boldsymbol{\psi}=-K \Delta \mathbf{n} \varphi$. Equation (12) turns into

$$
\begin{gathered}
\left.\frac{J}{2} \int\|\mathbf{v}\|^{2} \varphi d x_{1} d x_{2}\right|_{0} ^{t} \\
=\int\left(\frac{J}{2}\left(\varphi_{t}+u^{j} \varphi_{x_{j}}\right)\|\mathbf{v}\|^{2}-K(\Delta \mathbf{n} \times \mathbf{n}) \cdot \mathbf{v} \varphi\right) d x_{1} d x_{2} d t
\end{gathered}
$$

$$
\begin{gathered}
\left.\int \frac{K}{2}\|\nabla \mathbf{n}\|^{2} \varphi d x_{1} d x_{2}\right|_{0} ^{t} \\
=\int\left(\frac{K}{2}\left(\varphi_{t}+u^{j} \varphi_{x_{j}}\right)\|\nabla \mathbf{n}\|^{2}-K(\mathbf{v} \times \mathbf{n}) \cdot \Delta \mathbf{n} \varphi\right. \\
\left.-K u_{x_{k}}^{j} \mathbf{n}_{x_{j}} \cdot \mathbf{n}_{x_{k}} \varphi-K \dot{\mathbf{n}}-\mathbf{n}_{x_{j}} \varphi_{x_{j}}\right) d x_{1} d x_{2} d t .
\end{gathered}
$$

Add the previous two identities.

$$
\begin{gathered}
\left.\frac{J}{2} \int\|\mathbf{v}\|^{2} \varphi d x_{1} d x_{2}\right|_{0} ^{t}+\left.\int \frac{K}{2}\|\nabla \mathbf{n}\|^{2} \varphi d x_{1} d x_{2}\right|_{0} ^{t} \\
\leq \int \dot{\varphi}\left(\frac{J}{2}\|\mathbf{v}\|^{2}+\frac{K}{2}\|\nabla \mathbf{n}\|^{2}\right)+K \max _{i, j, x} w^{i j}\|\nabla \mathbf{n}\|^{2} \varphi \\
+K\|\mathbf{v}\|\|\nabla \mathbf{n}\|\|\nabla \varphi\| d x_{1} d x_{2} d t .
\end{gathered}
$$

Denote $\varphi=\phi\left(\|x\|+m^{\prime} t\right)$, where $\phi(x) \in C^{1}(\mathbb{R}), \phi=0$ for $\|x\|>r, \phi^{\prime} \leq 0$. Let $m^{\prime}:=m+\max \left\{1, \frac{K}{J}\right\}$ and estimate

$$
\begin{gathered}
\int\left(\dot{\varphi}\left(\frac{J}{2 K}\|\mathbf{v}\|^{2}+\frac{1}{2}\|\nabla \mathbf{n}\|^{2}\right)+\|\mathbf{v}\|\|\nabla \mathbf{n}\|\|\nabla \varphi\|\right) d x_{1} d x_{2} d t \\
\leq \int \phi^{\prime}\left(\|x\|+m^{\prime} t\right)\left(m^{\prime}+\frac{u^{i} x_{i}}{\|x\|}\right)\left(\frac{J}{2 K}\|\mathbf{v}\|^{2}+\frac{1}{2}\|\nabla \mathbf{n}\|^{2}\right) \\
+\frac{\left|\phi^{\prime}\left(\|x\|+m^{\prime} t\right)\right|}{2}\left(\|\mathbf{v}\|^{2}+\|\nabla \mathbf{n}\|^{2}\right) d x_{1} d x_{2} d t \leq 0 .
\end{gathered}
$$

Consequently,

$$
\begin{aligned}
& \frac{J}{2 K}\left\|\mathbf{v}(t) \phi^{\frac{1}{2}}\right\|_{2}^{2}+\frac{1}{2}\left\|\phi^{\frac{1}{2}} \nabla \mathbf{n}(t)\right\|_{2}^{2} \\
& \leq \int_{i, j, x} \max ^{i j}\|\nabla \mathbf{n}\|^{2} \varphi d x_{1} d x_{2} d t \\
& \leq M t^{\frac{\alpha-1}{\alpha}} \text { ess } \sup _{t}\left\|\phi^{\frac{1}{2}} \nabla \mathbf{n}\right\|_{2}^{2},
\end{aligned}
$$

which proves the statement.

Remark 2. The proof of Theorem 4 is independent of the existence and uniqueness proof and result. Moreover, in the proof we can suppose $(\mathbf{u}, \mathbf{v}, \mathbf{n})$ to satisfy only (3), (4) but not (2).

\section{ACKNOWLEDGMENTS}

The second and the third authors was partially supported by RFBR grant 15-01-00838, the fourth author was partially supported by RFBR grant 15-01-07920. The first author was partially supported by Swiss NSF grant 200021-140238. 


\section{REFERENCES}

1. F. H. Lin, Commun. Pure Appl. Math. 42, 789-814 (1989).

2. F. H. Lin and C. Liu, Commun. Pure Appl. Math. 47, 501-537 (1995).

3. S. Shkoller, Commun. Part. Differ. Equations 27, 1103-1137 (2002).

4. M. C. Hong, Calc. Var. Part. Differ. Equations 40, 1536 (2011).

5. F. H. Lin, J. Y. Liu, and C. Y. Wang, Arch. Ration. Mech. Anal. 197, 297-336 (2010).

6. G. A. Chechkin, T. S. Ratiu, M. S. Romanov, and V. N. Samokhin, Vestn. Mosk. Gos. Univ. Pechati 12, 139-151 (2012).

7. T. S. Ratiu, M. S. Romanov, and G. A. Chechkin, Probl. Mat. Anal. 66, 167-173 (2012).
8. J. Ericksen, Trans. Soc. Rheol. 5, 22-34 (1961).

9. F. Leslie, Quart. J. Mech. Appl. Math. 19, 357-370 (1966).

10. F. Gay-Balmaz and T. S. Ratiu, Adv. Appl. Math. 42, 176-275 (2009).

11. D. Forster, T. C. Lubensky, P. C. Martin, J. Swift, and P. S. Pershan, Phys. Rev. Lett. 29, 1016-1019 (1971).

12. O. A. Ladyzhenskaya and N. N. Uraltseva, Linear and Quasilinear Elliptic Equations (Academic, New York, 1968).

13. S. L. Sobolev, Some Applications of Functional Analysis in Mathematical Physics, 3rd ed. (Am. Math. Soc., Providence, R.I., 1991).

14. V. P. Mikhailov, Partial Differential Equations (Mir, Moscow, 1978). 